\title{
A data envelopment analysis approach for resource allocation with undesirable outputs: an application to home appliance production companies
}

\author{
MOHAMMAD NEMATI and REZA KAZEMI MATIN*(D) \\ Department of Mathematics, Karaj Branch, Islamic Azad University, Karaj, Iran \\ e-mail: Mohammad.Nemati@kiau.ac.ir; rkmatin@kiau.ac.ir
}

MS received 12 April 2018; revised 7 July 2018; accepted 4 August 2018; published online 2 January 2019

\begin{abstract}
Traditional data envelopment analysis (DEA) models use all multiple inputs and outputs to estimate efficiency scores of decision-making units (DMUs). Each unit may consist of several subunits in cases such as manufacturing systems, and each subunit may produce both desirable and undesirable outputs. Providing information about the proportion of resources for each subunit could assist managers in making better decisions for increasing the efficiency of production systems. The current study proposes a new approach for resource allocation and efficiency estimation of production units by considering partial impacts among inputs and outputs in the DEA framework. A weak disposable technology is used in these evaluations, and an empirical application of the proposed approach for obtaining performance of home appliances production companies is provided for illustration purposes.
\end{abstract}

Keywords. Data envelopment analysis (DEA); partial impacts; undesirable outputs; weak disposability; resource allocation.

\section{Introduction}

Data envelopment analysis (DEA) was introduced by Charnes et al [1]. DEA is a practical tool for evaluating the performance of peer decision-making units (DMUs) that use multiple inputs to produce multiple outputs. In general expression, the aim is to minimize input and maximize output in classical production theory methods and in particular the expression in DEA. As discussed in $\mathrm{Wu}$ et al [2], due to resource constraints and unlimited needs of the community, the issue of optimal allocation of resources is raised. Optimal allocation of resources is the most important tool for implementing a long-term strategy and plan in any organization. In other words, the policies and goals of each organization's program are reflected in the allocation of resources in an optimal manner. The amount of achievement depends on how resources are allocated and controlled. One of the important issues that should be considered in the production process is the optimal allocation of resources with undesirable factors. In the real world applications, there may be both desirable and undesirable outputs in production processes such as factories, hospitals, greenhouses or powerhouses where several departments and organizations work in conjunction. Desirable outputs may include health services, crops,

*For correspondence power, etc. and undesirable outputs could include aerosols, waste and pollution. In production theory literature, many authors considered undesirable outputs in the production processes (DMUs) and they presented many approaches for measuring the efficiency of DMUs by consideration the optimal allocation of resources. The concept of optimal allocation of resource can be studied in different dimensions.

In most of DEA approaches, the resources allocation is in such a way that all inputs are used completely to produce good outputs and bad outputs. For example, Hailu and Veeman [3] extended the Chavas-Cox approaches [4] to the non-parametric analysis by incorporating undesirable outputs and applied their model to measuring the efficiency of Canadian pulp and paper industry. Kuosmanen [5] presented a production technology based on weak disposability assumptions to calculate the environmental performance of units with undesirable outputs. Zhou et al [6] suggested a non-radial DEA approach to measure the environmental performance of OECD countries. Bain and Yang [7] developed several DEA models for measuring the aggregated efficiency of resource and environment based on environment DEA technology, which can be provided with an index to analyse DMU energy efficiencies and environmental efficiencies simultaneously. A centralized resource allocation (CRA) DEA method for accidental data, namely SCRA model, was suggested by Hosseinzadeh Lotfi et al 
[8]. Their approach creates a stochastic DEA structure for CRA. Using their method, accidental propositions are transformed into decisive and analysable propositions. Then by using the decisive CRA models, the accidental propositions can be converted into efficient ones. Mandal [9] coined the term "energy efficiency" to calculate the performance of Indian cement industry. Wu et al [2] proposed a DEA approach for allocation of the resource by considering bad outputs. In their approach, two objective functions are modelled, i.e., maximization of all good outputs and minimization all bad outputs. Dakpo et al [10] explained the recent developments on the inclusion of undesirable outputs in production technology modelling, namely material balance principles and weak G-disposability, by-product modelling and cost disposability assumptions. For further reference see Yan et al [11], Färe and Grosskopf [12], Basso and Pecati [13], Hadi-Vencheh et al [14], Cecilio and Diego [15], Gomes and Lins [16], Lin et al [17], Korhonen and Syrjanen [18] and Yang et al [19].

In the recent DEA literature, some other approaches such as network DEA methods and some other methods were proposed for measuring the efficiency of the production process by considering the optimal allocation of resource in the presence of undesirable outputs. Some of them are as follows. Amirteimoori et al [20] offered a two-stage network structure with undrainable outputs and applied their model to evaluate the performance of Green Hen poultry chains in the Guilan province, Iran. Ming-Miin [21] proposed a centralized network DEA model that merges the centralized DEA model and network DEA for the allocation of resources between subunits. His approach considers that in the circumstances in which bad outputs are commonly generated by good outputs, the decrease of bad outputs is related to the decrease of inputs, and some inputs are appropriated to the special subunit while some inputs are allocated between subunits. Fukuyama and Weber [22] offered a two-stage network structure for estimating the performance of DMUs by considering that inputs are consumed to create only final outputs and they applied their model for calculating the inefficiency of Japanese banks. Wu et al [23] suggested twostage network processes. In their approach, some of the resources are shared and resources are recovered by bad outputs. Also, the non-cooperative performance values are presented for the system. In addition, a heuristic algorithm is proposed for converting the nonlinear structure into a linear form. Mogale et al [24] proposed a two-stage model for transportation and allocation of food grain in the supply chain network structure in India.

The concept of resource allocation was also studied in supply chain management. Mogale et al [25] proposed a Mixed Integer Non-Linear Programming (MINLP) model for resource allocation. In their method, some decisions are taken for collecting the food grain in supply chain structure in India. Also, different operations like purchasing, collecting, transporting and distributing are considered in food grain supply chain of India. Their approach presents an
MINLP model for scheming in collecting food grain from surplus states to deficit states considering the seasonal procurement, silo capacity, demand satisfaction and vehicle capacity constraints. Mogale et al [26] presented a multiperiod inventory transporting method for tactical scheming of food grain supply chain. In their approach, an MINLP model is modelled, which asks for minimizing the overall cost comprising those of the total of food grain goods, collection and execution

In assessing the performance of industrial units and supply chain, knowledge of environmental factors such as undesirable outputs play an important role. By studying previous methods for performance evaluation of DMUs in the presence of undesirable outputs, it is found that resources are shared equally among both desirable and undesirable outputs in all models. However, it is possible that there is some partial impact between inputs and good outputs and bad outputs.

The current work attempts to estimate the percentage of each resource that is used by each production line without considering any network structure. Gauging the partial impacts of inputs on desirable and undesirable outputs has been attempted with regard to the recent work by Imanirad et al [27]. Weak disposability assumption is applied as proposed by Kuosmanen [5] to suggest a novel DEA approach for estimating the aggregate efficiency of DMUs by taking undesirable outputs into account. The model of Kuosmanen [5] is an important development in calculating the efficiency of DMUs with undesirable outputs and his method was also studied in the following papers: Yang and Pollitt [28], Wu et al [2], Kuosmanen and Podinovski [29].

Our proposed approach makes it possible to estimate efficiencies and to analyse performances of production systems with partial impacts among inputs and outputs when both desirable and undesirable outputs coexist. An empirical application of the proposed approach for performance evaluation of 60 home appliance production companies is also provided, which demonstrates the applicability of the new approach in practical applications. Some of the novelty points that distinguish the proposed approach in this article from those of previous models are as follows:

- for the first time, a new approach is suggested in this paper to calculate the aggregate efficiency of production units by considering bad outputs;

- in the new proposed model, the percent of resources (inputs) that lead to good output and bad output in each subunit is estimated;

- the current model can provide estimation of subunit efficiency in each unit;

- the overall efficiency is estimated by combining subunit efficiency.

The rest of the paper is organized as follows. Section 2 presents a brief review of weakly disposable technology and an example of resource allocation in traditional DEA 
models with undesirable outputs. A new DEA method is suggested for considering partial input-output impact in the presence of undesirable outputs in section 3 , and section 4 is devoted to presenting an application of the new method to home appliance production companies. Further discussion is presented in section 5 , and section 6 presents general conclusions and suggestions for future studies.

\section{DEA resource allocation with undesirable output}

A production process may include undesirable (bad) outputs as well as desirable (good) outputs. Several DEA models have been suggested for evaluating the performance of industrial units in the presence of undesirable outputs in the past decades. Kuosmanen [5] introduced a production technology based on the weakly disposable axiom of outputs for modelling undesirable outputs in DEA framework.

For a formal presentation, assume a set of $J$ DMUs to be evaluated in terms of a set of $I$ inputs $\left(x_{i}, i=1, \ldots, I\right), M$ good outputs $\left(v_{m}, m=1, \ldots, M\right)$ and $S$ bad outputs $\left(w_{s}, s=1, \ldots, S\right)$. Inputs and good outputs are assumed to be freely disposable under this setting. Desirable and undesirable outputs are weakly disposable and output set $\hat{P}(X)$ is assumed to be convex under a constant return to scale (CRS) assumption. The linear programming form of this weakly disposable technology for evaluating the performance of $\mathrm{DMU}_{o}$ is formulated as follows; for further reading see Kuosmanen and Kazemi Matin [30]:

$$
\max \sum_{m=1}^{M} u_{m} v_{m j_{o}}-\sum_{s=1}^{S} l_{s} w_{s j_{o}}
$$

subject to

$$
\begin{aligned}
& \sum_{m=1}^{M} u_{m} v_{m j}-\sum_{s=1}^{S} l_{s} w_{s j}-\sum_{i=1}^{I} d_{i} x_{i j} \leq 1 \quad j=1, \ldots, J \\
& \forall m, i: u_{m}, d_{i} \geq 0 \quad \forall s: l_{s} \text { free in sign. }
\end{aligned}
$$

Figure 1 shows the default of how inputs affect desirable and undesirable outputs when using Model (1) and all traditional DEA models including undesirable outputs. It is assumed that there are three inputs: two good outputs and one bad output, in each DMU.

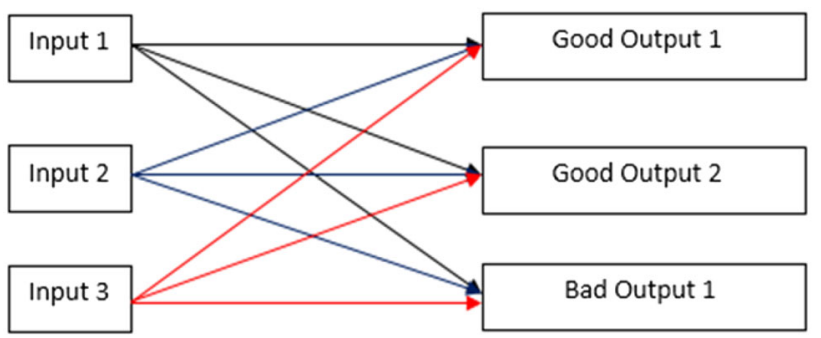

Figure 1. Impact of input on good and bad output in Model (1).
Note that all good and bad outputs are affected by all inputs in Model (1) as shown in figure 1. In other words, all inputs are shared equally among all good and bad outputs. It is possible to have situations where a unit has multiple subunits that use existing shared resources according to their needs in DEA applications. A simple example is used to illustrate this issue better.

\subsection{Motivating example}

Traditional models like Model (1) are useful for evaluating efficiency scores of DMUs where the entire output spectrum including both good and bad outputs is influenced by all inputs. Consider an industrial unit with two production lines as outputs. Undesirable outputs such as wasted products also coexist in the second production line. Therefore, managers should consider a recycling cost as input for waste products. Therefore, two inputs are considered in this evaluation: raw materials and recycling cost. In applying Model (1) for efficiency evaluation of units, the default interrelation between inputs and good and bad outputs is shown in table 1 and depicted in figure 2.

The following features of traditional DEA models such as Model (1) may be pointed out:

- as shown in figure 2, all good and bad outputs are assumed to be affected by all inputs, whereas recycling cost should have effects on only production line 2 ;

- provided efficiency score by Model (1) may underestimate the true efficiency score by considering the effect of recycling cost for both production lines.

As a result of these observations, it may be concluded that traditional DEA models are not able to calculate the correct efficiency of DMUs when partial impact between inputs and good and bad outputs exists.

Business units may have several production lines in practical applications where undesirable outputs affect only

Table 1. Interrelations between inputs and good and bad outputs in industrial units.

\begin{tabular}{lccc}
\hline & Production line 1 & \multicolumn{2}{c}{ Production line 2 } \\
\cline { 3 - 4 } DMU & Good output & Good output & Bad output \\
\hline Raw materials & $\checkmark$ & $\checkmark$ & $\checkmark$ \\
Recycling cost & $\checkmark$ & $\checkmark$ & $\checkmark$ \\
\hline
\end{tabular}

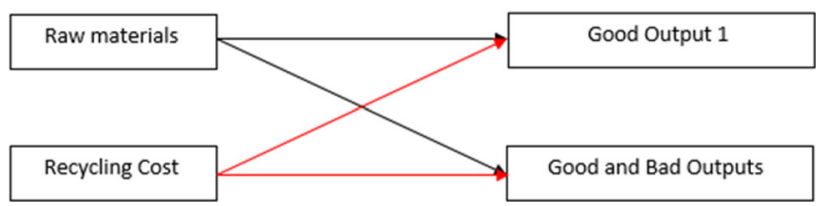

Figure 2. Default interrelations between inputs and good and bad outputs in industrial units. 
some inputs but not outputs. Standard DEA models need to be modified before application to performance assessment in such cases.

An innovative DEA model is presented in the next section to estimate the efficiency score that takes into account both issues of undesirable outputs and the existence of shared resource impacts.

\section{New proposed method}

\subsection{Structure of model}

A practical application is considered in this section with bad outputs to present the idea of partial input-output impact. In this evaluation, there are 60 home appliance firms, which include three production lines. These production lines include both good outputs and bad outputs:

i. Audio and video devices such as TVs, home theaters, camcorders, digital cameras, video projectors, etc.

ii. Heating and cooling devices such as air conditioners, fans, heaters, etc.

iii. Home appliance devices such as refrigerators, juicers, meat grinders, vacuum cleaners, cookers, washing machines, dishwashers, etc.

Furthermore, bad outputs may be considered as the number of waste byproducts that are present in each production line. For example, suppose there are 10 defective TVs or cameras out of each 100 assemblies in production line 1 . Resource inputs are considered as (1) firm labour, (2) packing machines, (3) pressing and (4) dyeing.

Table 2 shows the interrelationship between resources and production lines. As shown, not all production lines including

Table 2. Interrelation between production lines and resources.

\begin{tabular}{|c|c|c|c|}
\hline \multirow[b]{2}{*}{ Resources } & \multicolumn{3}{|c|}{ Production lines } \\
\hline & $\begin{array}{l}\text { Production } \\
\text { line } 1 \\
\text { Audio and } \\
\text { video } \\
\text { devices } \\
\text { (Including } \\
\text { Good } \\
\text { outputs) }\end{array}$ & $\begin{array}{l}\text { Production line } \\
\qquad 2 \\
\text { Heating and } \\
\text { cooling devices } \\
\text { (Including Good } \\
\text { and Bad } \\
\text { outputs) }\end{array}$ & $\begin{array}{c}\text { Production line } \\
\qquad 3 \\
\text { Home appliance } \\
\text { devices } \\
\text { (Including } \\
\text { Good and } \\
\text { Bad outputs) }\end{array}$ \\
\hline $\begin{array}{l}\text { Firm labour } \\
\quad \text { (input 1) }\end{array}$ & $\checkmark$ & $\checkmark$ & $\checkmark$ \\
\hline $\begin{array}{l}\text { Pressing } \\
\quad \text { (input 2) }\end{array}$ & $\checkmark$ & - & $\checkmark$ \\
\hline $\begin{array}{l}\text { Packing } \\
\text { machines } \\
\text { (input 3) }\end{array}$ & $\boldsymbol{V}$ & $\checkmark$ & $\checkmark$ \\
\hline $\begin{array}{l}\text { Dyeing } \\
\quad \text { (input 4) }\end{array}$ & $\boldsymbol{V}$ & - & $\checkmark$ \\
\hline
\end{tabular}

good and bad outputs are influenced by all resource inputs. In other words, each production line uses resources according to its needs. As an example, the usage of pressing may not be needed for producing heating and cooling devices, while pressing is required in the production of audio and video devices. The interrelation between resource inputs and production lines that include good and bad outputs could be classified as a bundle.

For formal presentation of the proposed model, suppose each firm contains $k$ subunits so that each subunit $k$ is represented by its own resource/production line bundle $\left(I_{k}, R_{k}\left(O_{G}, O_{B}\right)\right) \quad k=1, \ldots, K$. Resource inputs are numbered as 1, 2, 3 and 4 for firm labour, pressing, packing machines and dyeing, respectively. Additionally, production lines are numbered as 1, 2 and 3, for audio and video devices, heating and cooling devices and home appliance devices, respectively. Home appliances firms have 2 subunits or bundles $\left(I_{1}, R_{1}\left(O_{G}, O_{B}\right)\right), \quad\left(I_{2}, R_{2}\left(O_{G}, O_{B}\right)\right)$, where $I_{1}=$ $(1,2,3,4), I_{2}=(1,3)$ and $R_{1}\left(\left(1_{G}, \emptyset\right),\left(3_{G}, 3_{B}\right)\right), R_{2}\left(2_{G}\right.$, $\left.2_{B}\right)$. It is assumed that production line 1 does not have any bad output in bundle $R_{1}\left(\left(1_{G}, \emptyset\right),\left(3_{G}, 3_{B}\right)\right)$.

Notations " $G$ " and " $B$ " for definitions of "good output" and "bad output" in bundle $R_{k}\left(O_{G}, O_{B}\right)$ are used from this point on.

It is further assumed that inputs are divisible and can be allocated between subunits. Split variables $\beta_{i k}$ are defined for the amount of good and bad output usage from inputs, in the value $\beta_{i k} x_{i}$ where $\sum_{k} \beta_{i k}=1$. Figure 3 shows the types of resource allocation between good and bad outputs in production lines. An algorithm for creating maximal bundles in a general case is presented in the appendix.

A novel DEA approach is proposed for calculating the efficiency of DMUs in such situations and presented in the next section.

\subsection{A new proposed method}

A new model is suggested in this section for performance evaluation of home appliance companies including several production lines, subunits in the presence of undesirable outputs. The performances of DMUs are calculated in three steps: 1-aggregate efficiency, 2-subunit efficiency and 3 -overall efficiency. Following three steps are proposed for achieving this goal:

Step 1. Split variables $\beta_{i k}$ are defined for obtaining values for good and bad output usage from the input $i$ in each bundle $k$. The aggregate efficiency of DMUs is then evaluated.

Step 2. Efficiencies for each production line subunit are estimated using split variables $\beta_{i k}$.

Step 3. Overall efficiency of the business unit under evaluation is assessed.

- Step 1: Calculating aggregate efficiency

It is supposed that a DMU is the sum of several sections, and there are no economies or diseconomies of scope. Aggregate efficiency is a weighted average of $k$ subunit performances. 


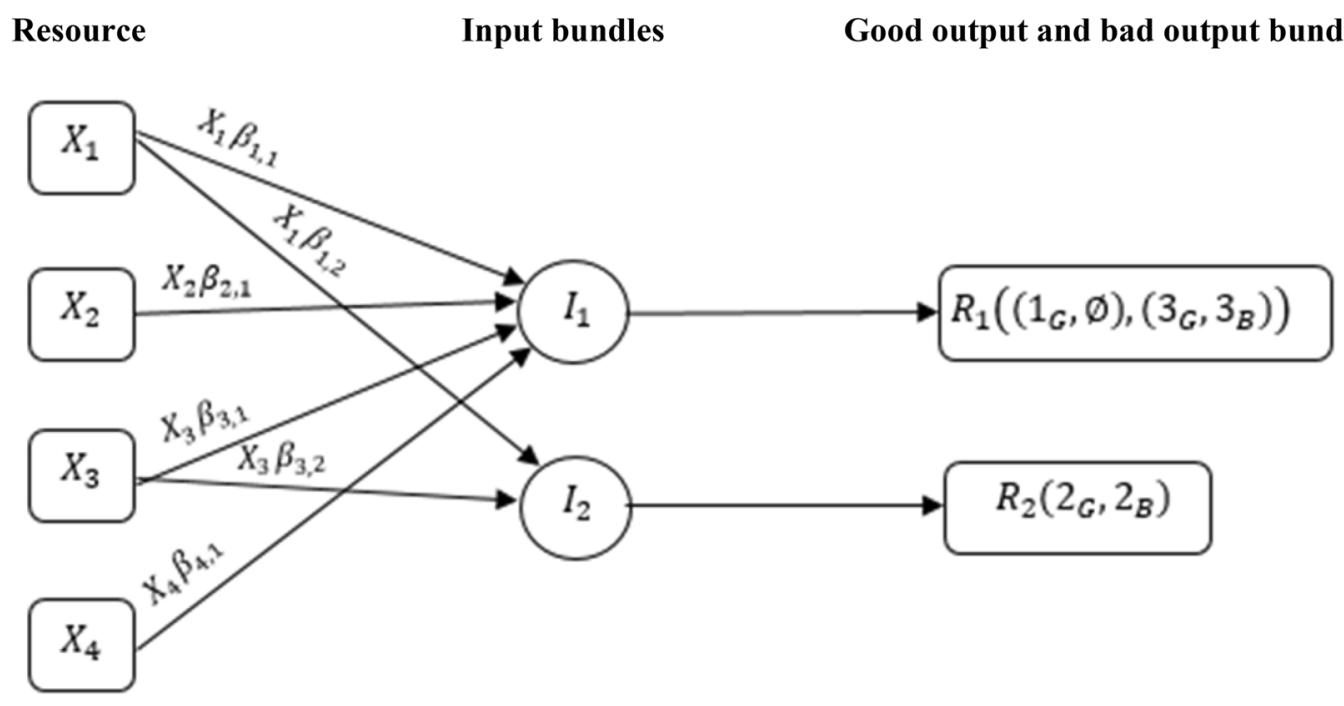

Figure 3. Resource allocation among subunits in the new model.

For this purpose, the CRS radial Model (1) is modified for deriving the $\beta$ - split of inputs with the objective of maximizing this aggregate efficiency with undesirable outputs:

$$
\begin{aligned}
& e_{a g g}=\max \\
& \sum_{k=1}^{K} H_{k j_{o}}\left[\frac{\sum_{m \in R_{k}\left(O_{G}, O_{B}\right)} u_{m} v_{m j_{o}}-\sum_{\left.s \in R_{k}\left(O_{G}, O_{B}\right)\right)} l_{s} w_{s j_{o}}}{\sum_{i \in I_{k}} d_{i} \beta_{i k} x_{i j_{o}}}\right]
\end{aligned}
$$

subject to

$\sum_{k=1}^{K} H_{k j}\left[\frac{\sum_{m \in R_{k}\left(O_{G}, O_{B}\right)} u_{m} v_{m j}-\sum_{s \in R_{k}\left(O_{G}, O_{B}\right)} l_{s} w_{s j}}{\sum_{i \in I_{k}} d_{i} \beta_{i k} x_{i j}}\right] \leq 1, \quad \forall j$

$\frac{\sum_{m \in R_{k}\left(O_{G}, O_{B}\right)} u_{m} v_{m j}-\sum_{s \in R_{k}\left(O_{G}, O_{B}\right)} l_{s} w_{s j}}{\sum_{i \in I_{k}} d_{i} \beta_{i k} x_{i j}} \leq 1, \quad \forall k, j$

$$
\begin{gathered}
\sum_{k \in T_{i}} \beta_{i k}=1, \quad \forall i \\
a_{i k} \leq \beta_{i k} \leq b_{i k}, \quad \forall i, k \\
u_{m}, d_{i}, \beta_{i k} \geq \varepsilon \quad \forall m, i, k \\
l_{s} \quad \text { free in sign } \quad \forall s .
\end{gathered}
$$

The notation $e_{a g g}$ is used and it indicates the mean "aggregate" efficiency score for the units under study. Further, constraints (2.2) defined for the variable $\beta_{i k}$ should be selected in such a way that the efficiency score of each subunit $k$ of DMU $j$ does not intrude on the unity of corresponding values of weights $u_{m}, d_{i}$ and $l_{s}$. Constraints (2.3) impose the convexity restriction on $\beta_{i k}$ values among subunit $k$ for each input $i$ that is exerted on those subunits. To insert restriction on the size of $\beta_{i k}$ variables, constraints (2.4) are imposed. Since there are weak disposability assumptions on undesirable outputs in constraints (2.6), variables $l_{s}$ are defined free of the sign.

Based on the modified Model (2), the weights $H_{k j}$ are defined as follows to indicate the significance of each subunit for DMU $j$ under consideration:

$$
H_{k j}=\sum_{i \in I_{k}} d_{i} \beta_{i k} x_{i j} / \sum_{k=1}^{K}\left[\sum_{i \in I_{k}} d_{i} \beta_{i k} x_{i j}\right] .
$$

Therefore, aggregate efficiency is represented as a convex combination of $k$ subunit efficiencies.

3.2a Linearization: Based on the definition of $H_{k j}$ as offered in Model (3), the objective function (2) may be rewritten as follows:

$$
\begin{aligned}
e_{0}= & \max \sum_{k=1}^{k} \sum_{m \in R_{k}\left(O_{G}, O_{B}\right)} u_{m} v_{m j_{o}} \\
& -\sum_{s \in R_{k}\left(O_{G}, O_{B}\right)} l_{s} w_{s j_{o}} / \sum_{i} d_{i} x_{i j_{o}} .
\end{aligned}
$$

In order to convert the present non-linear structure of Model (2) to a linear form, the variable substitution $z_{i k}=$ $d_{i} \beta_{i k}$ is used, and as a result $\sum_{k \in T_{i}} d_{i} \beta_{i k}=d_{i}$ is obtained, which may be rewritten as $\sum_{k \in T_{i}} z_{i k}=d_{i}$. Utilizing the standard Charnes and Cooper [31] transformation technique $t=$ $1 / \sum_{i} d_{i} x_{i j_{o}}$ and by defining $\mu_{m}=t v_{m}, \quad \rho_{i}=t d_{i}, \delta_{i k}=$ $t z_{i k}, \theta_{s}=t l_{s}$, Model (2) is converted to the following linear programming form: 


$$
e_{a g g}=\max \sum_{k=1}^{K}\left[\sum_{m \in R_{k}\left(O_{G}, O_{B}\right)} \mu_{m} v_{m j_{o}}-\sum_{s \in R_{k}\left(O_{G}, O_{B}\right)} l_{s} w_{s j_{o}}\right]
$$

$$
\begin{gathered}
\text { subject to } \\
\sum_{i} \rho_{i} x_{i j_{o}}=1, \\
\sum_{m \in R_{k}\left(O_{G}, O_{B}\right)} \mu_{m} v_{m j}-\sum_{s \in R_{k}\left(O_{G}, O_{B}\right)} \theta_{s} w_{s j}-\sum_{i \in I_{k}} \delta_{i k} x_{i j} \leq 0, \quad \forall j, k
\end{gathered}
$$

$$
\begin{gathered}
\sum_{k \in T_{i}} \delta_{i k}=\rho_{i}, \quad \forall i \\
\rho_{i} a_{i k} \leq \delta_{i k} \leq \rho_{i} b_{i k}, \quad \forall i, k \\
\mu_{m}, \rho_{i}, \delta_{i k} \geq \varepsilon \quad \forall m, i, k \\
\theta_{s} \quad \text { free in sign } \quad \forall s .
\end{gathered}
$$

Note that the set $T_{i}$ in restrictions (4.3) is defined as the set of all subunits $k$ that have $i$ as a member. For example, as shown in figure 2, input $X_{2}$ is allocated only to subunit 1; therefore, the weights $\delta_{2,1}$ and $d_{2}$ are equivalent. Since $\delta_{2,1}$ is limited as $d_{2} a_{2,1} \leq \delta_{2,1} \leq d_{2} b_{2,1}$ in restriction (4.4), this restriction becomes redundant.

- Step 2: Calculating subunit efficiency score

One of the key results from the solution of Model (4) is the resource split-variable $\beta_{i k}$. Note that $\beta_{i k}=\delta_{i k} / \rho_{i}$. Some of the production lines follow bad outputs. Therefore, the efficiency score may be derived by considering that each bundle output uses an appropriate portion of inputs $\left(\beta_{i k} x_{i j}\right)$ according to its respective production line subunits. This model makes a valuable contribution to theoretical DEA literature by demonstrating how resources are allocated when the partial impact of input to good output and bad output exists. Finally, Model (2) is applied with the knowledge that the evaluated "DMU" is the $k^{\text {th }}$ subunit where its good outputs and bad outputs are the members of $R_{k}$ in the amounts $v_{m j}$ and $w_{s j}$, and its inputs are the members of $I_{k}$ in amounts $\beta_{i k} x_{i j}$.

- Step 3: Obtaining the overall efficiency score

To calculate the overall efficiency scores $e_{\text {ove }}$ of the $D M U$ s, the weighted average of the $k$ subunit scores that were obtained in stage 2 is needed while using $H_{k j}$ defined in (3). In order to compute $H_{k j}$, a suitable set of input multipliers $d_{i}$ must be chosen. These multipliers should be calculated under the conditions where all subunits are being compared similarly. The aggregate Model (4) offers such a condition. If $D M U_{j_{o}}$ is to be evaluated, the input portion of constraint (4.2), i.e., $\sum_{i \in I_{k}} \delta_{i k} x_{i j}$, represents the amount of
DMU resources that is attributed to subunit $k$. The sum of all consumed resources by $D M U_{j_{o}}$ is shown by $\sum_{i} \rho_{i} x_{i j_{o}}$, which is naturally scaled to unity by constraint (4.1). Therefore, the weights $H_{k j}$ can be written as $H_{k j}=\sum_{i \in I_{k}} \delta_{i k} x_{i j}$. Weights $H_{k j}$ are used to obtain the overall efficiency scores for the DMUs.

\section{Illustrative application and discussions}

\subsection{Illustrative application}

In this section, the proposed approach is applied to performance measurement of 60 home appliance production companies in Iran. Collected data ${ }^{1}$ are reported in table 3.

- Calculating aggregate efficiency

Aggregate efficiency is calculated in the interval $\left[a_{i k}, b_{i k}\right]=[0.3,0.9]$ by Model (4) and results are presented in table 4. For example, to calculate the aggregate efficiency of DMU 60 we need to solve the following linear programming optimization model:

$\max 365 \mu_{1}+18731 \mu_{3}-1151 \theta_{2}+2088 \mu_{2}-0$

subject to

$$
\begin{aligned}
& 452 \rho_{1}+18.97 \rho_{2}+13.17 \rho_{3}+1.114 \rho_{4}=1 \\
& 1783 \mu_{1}+12854 \mu_{3}-1155 \theta_{2}-270 \delta_{1,1} \\
& -10.1 \delta_{2,1}-10 \delta_{3,1}-1.2 \delta_{4,1}
\end{aligned}
$$$$
\leq 0 \quad(\text { subunit } 1 \text { DMU } 1)
$$

subunit 1 DMU $2, \ldots$, subunit 1 DMU 59

$2086 \mu_{1}+18731 \mu_{3}-1151 \theta_{2}-452 \delta_{1,1}-18.97 \delta_{2,1}$

$-13.17 \delta_{3,1}-1.114 \delta_{4,1} \leq 0$

(subunit 1 DMU 60)

$412 \mu_{1}-30 \theta_{1}-270 \delta_{1,2}-10 \delta_{3,2} \leq 0 \quad$ (subunit 2 DMU 1$)$

subunit 2 DMU 2,.., subunit 2 DMU 59

$356 \mu_{1}-0 \theta_{1}-452 \delta_{1,2}-13.17 \delta_{3,2} \leq 0$

(subunit 2 DMU 60)

$\delta_{1,1}+\delta_{1,2}=\rho_{1} \quad \delta_{2,1}=\rho_{2} \quad \delta_{3,1}+\delta_{3,2}=\rho_{3}$

$\delta_{4,1}=\rho_{4} \quad \delta_{1,1} \geq 0.3 \rho_{1}$

$\delta_{1,2} \geq 0.3 \rho_{1} \quad \delta_{3,1} \geq 0.3 \rho_{3} \quad \delta_{3,2} \geq 0.3 \rho_{3} \quad \delta_{1,1} \leq 0.9 \rho_{1}$

$\delta_{1,2} \leq 0.9 \rho_{1} \delta_{3,2} \leq 0.9 \rho_{3} \quad \delta_{3,1} \leq 0.9 \rho_{3}$

$\rho_{1} \ldots \rho_{4}, \delta_{1,1}, \delta_{1,2}, \delta_{3,1}, \delta_{3,2}, \mu_{1}, \mu_{2}, \mu_{3} \geq \varepsilon=0.00001$,

free in $\operatorname{sign} \theta_{1}, \theta_{2}$.

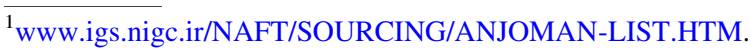


Table 3. Input-output data for 60 firms.

\begin{tabular}{|c|c|c|c|c|c|c|c|c|c|}
\hline \multirow[b]{3}{*}{ DMU } & \multicolumn{4}{|c|}{ Inputs } & \multicolumn{5}{|c|}{ Production lines } \\
\hline & \multirow{2}{*}{$\begin{array}{c}\text { Labour } \\
\quad X 1\end{array}$} & \multirow{2}{*}{$\begin{array}{c}\text { Pressing } \\
\quad X 2\end{array}$} & \multirow{2}{*}{$\begin{array}{c}\text { Packing } \\
X 3\end{array}$} & \multirow{2}{*}{$\begin{array}{c}\text { Dyeing } \\
X 4\end{array}$} & \multicolumn{3}{|c|}{ Good outputs } & \multicolumn{2}{|c|}{ Bad outputs } \\
\hline & & & & & $V 1$ & $V 2$ & $V 3$ & $W 1$ & $W 2$ \\
\hline 1 & 270 & 10.1 & 10 & 1.2 & 1783 & 412 & 12854 & 30 & 1155 \\
\hline 2 & 260 & 14.3 & 19 & 0.7 & 2266 & 765 & 24673 & 40 & 2164 \\
\hline 3 & 350 & 21.4 & 10.5 & 0.8 & 6872 & 298 & 43525 & 35 & 2342 \\
\hline 4 & 380 & 18.4 & 11 & 0.9 & 2372 & 176 & 93184 & 38 & 5346 \\
\hline 5 & 156 & 12.3 & 16.1 & 0.5 & 2763 & 287 & 21286 & 28 & 1155 \\
\hline 6 & 140 & 9.3 & 12.2 & 1.6 & 6234 & 276 & 31125 & 37 & 2338 \\
\hline 7 & 170 & 18 & 20.2 & 2.1 & 3454 & 762 & 42986 & 31 & 3441 \\
\hline 8 & 285 & 19 & 18.6 & 1.6 & 3324 & 871 & 63437 & 1 & 6315 \\
\hline 9 & 285 & 16 & 12.5 & 1.45 & 6369 & 655 & 42872 & 25 & 4248 \\
\hline 10 & 160 & 11 & 17.8 & 1.15 & 7662 & 297 & 64476 & 1 & 6132 \\
\hline 11 & 280 & 11.7 & 12.2 & 1.15 & 5632 & 546 & 42235 & 25 & 4414 \\
\hline 12 & 332 & 13.5 & 18.9 & 0.64 & 4577 & 231 & 12245 & 1 & 1233 \\
\hline 13 & 228 & 14.7 & 15.7 & 1.09 & 3545 & 264 & 27287 & 1 & 2126 \\
\hline 14 & 248 & 16.8 & 14.6 & 0.99 & 4852 & 463 & 64674 & 32 & 6134 \\
\hline 15 & 179 & 12.3 & 22.7 & 1.22 & 8762 & 582 & 54740 & 26 & 5343 \\
\hline 16 & 299 & 11.4 & 18.9 & 0.49 & 8983 & 398 & 32492 & 1 & 3142 \\
\hline 17 & 257 & 10.1 & 18.88 & 0.55 & 5786 & 365 & 21481 & 25 & 2523 \\
\hline 18 & 355 & 25.5 & 13.2 & 1.16 & 2634 & 873 & 43265 & 32 & 4528 \\
\hline 19 & 220 & 19.7 & 14.2 & 1.68 & 7281 & 547 & 34764 & 33 & 3439 \\
\hline 20 & 197 & 15.8 & 20.9 & 1.22 & 6491 & 325 & 11461 & 27 & 1343 \\
\hline 21 & 125 & 12.3 & 14.2 & 0.93 & 1254 & 287 & 35296 & 10 & 3123 \\
\hline 22 & 167 & 11.5 & 10.4 & 1.21 & 3421 & 542 & 27548 & 1 & 2658 \\
\hline 23 & 145 & 13.6 & 11.3 & 1.7 & 8723 & 358 & 87354 & 12 & 8456 \\
\hline 24 & 165 & 15.5 & 12.8 & 0.34 & 1254 & 673 & 23461 & 32 & 2428 \\
\hline 25 & 172 & 16.8 & 16.8 & 0.13 & 1367 & 982 & 87354 & 39 & 8763 \\
\hline 26 & 187 & 21.4 & 21.5 & 0.983 & 1366 & 283 & 45268 & 25 & 4254 \\
\hline 27 & 129 & 18.4 & 17.9 & 0.543 & 7623 & 126 & 43286 & 98 & 4643 \\
\hline 28 & 231 & 23.6 & 18.3 & 0.429 & 2876 & 284 & 54628 & 4 & 5528 \\
\hline 29 & 248 & 10.4 & 14.9 & 0.284 & 3523 & 339 & 32671 & 32 & 3428 \\
\hline 30 & 321 & 14.6 & 23.6 & 0.183 & 3790 & 397 & 67298 & 22 & 5762 \\
\hline 31 & 362 & 15.6 & 11.8 & 0.329 & 6732 & 653 & 37519 & 45 & 3163 \\
\hline 32 & 183 & 13.5 & 17.6 & 0.678 & 5432 & 473 & 28745 & 1 & 2983 \\
\hline 33 & 193 & 16.7 & 18.4 & 0.382 & 8715 & 459 & 27546 & 63 & 2572 \\
\hline 34 & 159 & 18.9 & 15.8 & 0.983 & 5629 & 256 & 87456 & 12 & 4467 \\
\hline 35 & 219 & 14.6 & 21.4 & 0.632 & 3891 & 334 & 58563 & 13 & 4863 \\
\hline 36 & 264 & 23 & 19 & 0.829 & 3764 & 654 & 46839 & 16 & 3537 \\
\hline 37 & 251 & 17 & 15 & 1.5 & 8743 & 562 & 28303 & 1 & 4729 \\
\hline 38 & 182 & 16.4 & 16.3 & 1.59 & 3454 & 238 & 35520 & 32 & 2452 \\
\hline 39 & 143 & 12.6 & 19.3 & 1.63 & 4982 & 783 & 38760 & 25 & 5562 \\
\hline 40 & 128 & 10.7 & 12.5 & 2.3 & 7652 & 712 & 46598 & 22 & 3427 \\
\hline 41 & 342 & 13.6 & 13.9 & 2.1 & 4328 & 831 & 65790 & 12 & 4271 \\
\hline 42 & 295 & 12.4 & 18.2 & 1.73 & 6712 & 432 & 87220 & 1 & 2528 \\
\hline 43 & 234 & 16.4 & 13.8 & 1.234 & 5639 & 901 & 64830 & 74 & 1173 \\
\hline 44 & 260 & 14.4 & 14.2 & 1.821 & 3519 & 619 & 23489 & 33 & 3352 \\
\hline 45 & 237 & 13.3 & 18.2 & 1.438 & 7645 & 563 & 56382 & 27 & 4278 \\
\hline 46 & 138 & 18 & 12 & 1.292 & 8632 & 198 & 36581 & 0 & 3753 \\
\hline 47 & 276 & 14 & 10.3 & 1.573 & 4529 & 231 & 47891 & 15 & 3563 \\
\hline 48 & 249 & 19 & 15 & 1.629 & 3562 & 100 & 32980 & 10 & 3825 \\
\hline 49 & 176 & 19.5 & 17.3 & 1.392 & 2382 & 173 & 76830 & 1 & 2476 \\
\hline 50 & 147 & 20 & 13.9 & 1.733 & 1853 & 351 & 77652 & 23 & 2387 \\
\hline 51 & 432 & 17.2 & 12.01 & 1.324 & 6822 & 406 & 75376 & 14 & 1633 \\
\hline 52 & 342 & 10.85 & 10.69 & 1.945 & 1460 & 819 & 69546 & 67 & 2687 \\
\hline 53 & 612 & 19.24 & 13.73 & 0.536 & 2789 & 412 & 40991 & 34 & 2496 \\
\hline 54 & 213 & 14.67 & 10.33 & 0.443 & 1540 & 963 & 82577 & 52 & 3623 \\
\hline
\end{tabular}


Table 3 continued

\begin{tabular}{|c|c|c|c|c|c|c|c|c|c|}
\hline \multirow[b]{3}{*}{ DMU } & \multicolumn{4}{|c|}{ Inputs } & \multicolumn{5}{|c|}{ Production lines } \\
\hline & \multirow{2}{*}{$\begin{array}{c}\text { Labour } \\
X 1\end{array}$} & \multirow{2}{*}{$\begin{array}{c}\text { Pressing } \\
\quad X 2\end{array}$} & \multirow{2}{*}{$\begin{array}{c}\text { Packing } \\
\text { X3 }\end{array}$} & \multirow{2}{*}{$\begin{array}{c}\text { Dyeing } \\
X 4\end{array}$} & \multicolumn{3}{|c|}{ Good outputs } & \multicolumn{2}{|c|}{ Bad outputs } \\
\hline & & & & & $V 1$ & $V 2$ & $V 3$ & $W 1$ & $W 2$ \\
\hline 55 & 763 & 14.54 & 12.96 & 0.8 & 7602 & 747 & 63896 & 49 & 3131 \\
\hline 56 & 692 & 10.18 & 17.82 & 1.315 & 9866 & 328 & 81342 & 10 & 2526 \\
\hline 57 & 319 & 12.84 & 12.95 & 1.499 & 4829 & 760 & 14562 & 29 & 1120 \\
\hline 58 & 476 & 12.57 & 16.94 & 1.347 & 6450 & 265 & 83896 & 60 & 5461 \\
\hline 59 & 936 & 16.07 & 12.46 & 1.419 & 3395 & 382 & 48031 & 30 & 5589 \\
\hline 60 & 452 & 18.97 & 13.17 & 1.114 & 2086 & 356 & 18731 & 1 & 1151 \\
\hline
\end{tabular}

Table 4. Efficiency scores resulting from Model (1) and Model (4).

\begin{tabular}{|c|c|c|c|c|c|}
\hline DMU & $\begin{array}{c}\text { Efficiency scores } \\
\text { Model (1) }\end{array}$ & $\begin{array}{c}\text { Aggregate efficiency scores } \\
\text { Model (4) }\end{array}$ & DMU & $\begin{array}{l}\text { Efficiency scores } \\
\text { Model (1) }\end{array}$ & $\begin{array}{c}\text { Aggregate efficiency scores } \\
\text { Model (4) }\end{array}$ \\
\hline 1 & 1.0000 & 0.4770 & 31 & 1.0000 & 0.9925 \\
\hline 2 & 1.0000 & 0.4904 & 32 & 0.8230 & 0.7366 \\
\hline 3 & 1.0000 & 0.9095 & 33 & 1.0000 & 0.9996 \\
\hline 4 & 1.0000 & 0.9070 & 34 & 1.0000 & 0.9640 \\
\hline 5 & 1.0000 & 0.5303 & 35 & 0.7761 & 0.7424 \\
\hline 6 & 1.0000 & 0.9563 & 36 & 1.0000 & 0.5550 \\
\hline 7 & 0.8767 & 0.6831 & 37 & 1.0000 & 0.8218 \\
\hline 8 & 0.7604 & 0.8407 & 38 & 0.4824 & 0.4241 \\
\hline 9 & 0.9348 & 0.7048 & 39 & 1.0000 & 0.8676 \\
\hline 10 & 1.0000 & 0.9998 & 40 & 1.0000 & 1.0000 \\
\hline 11 & 0.9411 & 0.7161 & 41 & 1.0000 & 0.9615 \\
\hline 12 & 0.6743 & 0.6375 & 42 & 1.0000 & 0.9995 \\
\hline 13 & 0.4608 & 0.4084 & 43 & 1.0000 & 1.0000 \\
\hline 14 & 0.7650 & 0.7107 & 44 & 1.0000 & 0.4454 \\
\hline 15 & 1.0000 & 0.9826 & 45 & 0.9156 & 0.8056 \\
\hline 16 & 1.0000 & 0.9994 & 46 & 1.0000 & 0.9168 \\
\hline 17 & 0.8519 & 0.7459 & 47 & 1.0000 & 0.5508 \\
\hline 18 & 1.0000 & 0.8316 & 48 & 0.4202 & 0.3546 \\
\hline 19 & 0.8498 & 0.6702 & 49 & 0.9954 & 0.8781 \\
\hline 20 & 1.0000 & 0.9993 & 50 & 1.0000 & 0.9399 \\
\hline 21 & 0.6589 & 0.4886 & 51 & 1.0000 & 0.9994 \\
\hline 22 & 0.8052 & 0.9390 & 52 & 1.0000 & 1.0000 \\
\hline 23 & 1.0000 & 0.9997 & 53 & 0.8690 & 0.5084 \\
\hline 24 & 1.0000 & 0.6609 & 54 & 1.0000 & 1.0000 \\
\hline 25 & 1.0000 & 1.0000 & 55 & 1.0000 & 0.9697 \\
\hline 26 & 0.6409 & 0.4158 & 56 & 1.0000 & 0.9989 \\
\hline 27 & 1.0000 & 1.0000 & 57 & 1.0000 & 0.7453 \\
\hline 28 & 0.6855 & 0.5549 & 58 & 0.9915 & 0.9671 \\
\hline 29 & 0.8803 & 0.7975 & 59 & 0.7725 & 0.5780 \\
\hline 30 & 1.0000 & 0.9991 & 60 & 0.4835 & 0.4879 \\
\hline
\end{tabular}

- Calculating production line (subunit) efficiency

Values of split variables $\beta_{i k}$ are obtained from Model (4) for calculating production line efficiencies. These values are then multiplied in inputs according to each good and bad output usage from inputs. Model (2) is finally applied, and results are collected in table 7 . Split variables $\beta_{i k}$ provide appropriate inputs, i.e., $\left(\beta_{i k} x_{i j}\right)$ to their respective production line. The values of split variables $\beta_{i k}$ are reported in table 5.

- Calculating overall efficiency scores

The $H_{k j}$ values are computed from Model (4) for two subunits $k=1,2$, where $H_{k j}=\sum_{i \in I_{k}} \delta_{i k} x_{i j}$. Results are 
Table 5. $\beta_{i k}$ values resulting from Model (4).

\begin{tabular}{|c|c|c|c|c|c|c|c|c|c|c|c|c|c|}
\hline DMU & $\beta_{1,1}$ & $\beta_{1,2}$ & $\beta_{2,1}$ & $\beta_{3,1}$ & $\beta_{3,2}$ & $\beta_{4,1}$ & DMU & $\beta_{1,1}$ & $\beta_{1,2}$ & $\beta_{2,1}$ & $\beta_{3,1}$ & $\beta_{3,2}$ & $\beta_{4,1}$ \\
\hline 1 & 0.3 & 0.7 & 1 & 0.1 & 0.9 & 1 & 31 & 0.5 & 0.5 & 1 & 0.7 & 0.3 & 1 \\
\hline 2 & 0.3 & 0.7 & 1 & 0.1 & 0.9 & 1 & 32 & 0.3 & 0.7 & 1 & 0.5 & 0.5 & 1 \\
\hline 3 & 0.7 & 0.3 & 1 & 0.7 & 0.3 & 1 & 33 & 0.7 & 0.3 & 1 & 0.1 & 0.9 & 1 \\
\hline 4 & 0.5 & 0.5 & 1 & 0.7 & 0.3 & 1 & 34 & 0.7 & 0.3 & 1 & 0.7 & 0.3 & 1 \\
\hline 5 & 0.7 & 0.3 & 1 & 0.5 & 0.5 & 1 & 35 & 0.7 & 0.3 & 1 & 0.5 & 0.5 & 1 \\
\hline 6 & 0.7 & 0.3 & 1 & 0.7 & 0.3 & 1 & 36 & 0.3 & 0.7 & 1 & 0.3 & 0.7 & 1 \\
\hline 7 & 0.3 & 0.7 & 1 & 0.5 & 0.5 & 1 & 37 & 0.7 & 0.3 & 1 & 0.7 & 0.3 & 1 \\
\hline 8 & 0.3 & 0.7 & 1 & 0.1 & 0.9 & 1 & 38 & 0.7 & 0.3 & 1 & 0.3 & 0.7 & 1 \\
\hline 9 & 0.5 & 0.5 & 1 & 0.7 & 0.3 & 1 & 39 & 0.3 & 0.7 & 1 & 0.5 & 0.5 & 1 \\
\hline 10 & 0.7 & 0.3 & 1 & 0.7 & 0.3 & 1 & 40 & 0.7 & 0.3 & 1 & 0.7 & 0.3 & 1 \\
\hline 11 & 0.5 & 0.5 & 1 & 0.7 & 0.3 & 1 & 41 & 0.3 & 0.7 & 1 & 0.1 & 0.9 & 1 \\
\hline 12 & 0.5 & 0.5 & 1 & 0.1 & 0.9 & 1 & 42 & 0.7 & 0.3 & 1 & 0.1 & 0.9 & 1 \\
\hline 13 & 0.7 & 0.3 & 1 & 0.7 & 0.3 & 1 & 43 & 0.7 & 0.3 & 1 & 0.1 & 0.9 & 1 \\
\hline 14 & 0.5 & 0.5 & 1 & 0.7 & 0.3 & 1 & 44 & 0.3 & 0.7 & 1 & 0.1 & 0.9 & 1 \\
\hline 15 & 0.7 & 0.3 & 1 & 0.5 & 0.5 & 1 & 45 & 0.7 & 0.3 & 1 & 0.7 & 0.3 & 1 \\
\hline 16 & 0.7 & 0.3 & 1 & 0.5 & 0.5 & 1 & 46 & 0.7 & 0.3 & 1 & 0.7 & 0.3 & 1 \\
\hline 17 & 0.4 & 0.6 & 1 & 0.1 & 0.9 & 1 & 47 & 0.5 & 0.5 & 1 & 0.7 & 0.3 & 1 \\
\hline 18 & 0.5 & 0.5 & 1 & 0.1 & 0.9 & 1 & 48 & 0.5 & 0.5 & 1 & 0.7 & 0.3 & 1 \\
\hline 19 & 0.7 & 0.3 & 1 & 0.7 & 0.3 & 1 & 49 & 0.7 & 0.3 & 1 & 0.5 & 0.5 & 1 \\
\hline 20 & 0.7 & 0.3 & 1 & 0.5 & 0.5 & 1 & 50 & 0.7 & 0.3 & 1 & 0.7 & 0.3 & 1 \\
\hline 21 & 0.7 & 0.3 & 1 & 0.5 & 0.5 & 1 & 51 & 0.5 & 0.5 & 1 & 0.1 & 0.9 & 1 \\
\hline 22 & 0.3 & 0.7 & 1 & 0.1 & 0.9 & 1 & 52 & 0.5 & 0.5 & 1 & 0.7 & 0.3 & 1 \\
\hline 23 & 0.7 & 0.3 & 1 & 0.1 & 0.9 & 1 & 53 & 0.5 & 0.5 & 1 & 0.7 & 0.3 & 1 \\
\hline 24 & 0.3 & 0.7 & 1 & 0.1 & 0.9 & 1 & 54 & 0.5 & 0.5 & 1 & 0.1 & 0.9 & 1 \\
\hline 25 & 0.3 & 0.7 & 1 & 0.7 & 0.3 & 1 & 55 & 0.5 & 0.5 & 1 & 0.7 & 0.3 & 1 \\
\hline 26 & 0.7 & 0.3 & 1 & 0.5 & 0.5 & 1 & 56 & 0.7 & 0.3 & 1 & 0.7 & 0.3 & 1 \\
\hline 27 & 0.3 & 0.7 & 1 & 0.5 & 0.5 & 1 & 57 & 0.7 & 0.3 & 1 & 0.7 & 0.3 & 1 \\
\hline 28 & 0.7 & 0.3 & 1 & 0.7 & 0.3 & 1 & 58 & 0.5 & 0.5 & 1 & 0.1 & 0.9 & 1 \\
\hline 29 & 0.4 & 0.6 & 1 & 0.1 & 0.9 & 1 & 59 & 0.5 & 0.5 & 1 & 0.7 & 0.3 & 1 \\
\hline 30 & 0.4 & 0.6 & 1 & 0.1 & 0.9 & 1 & 60 & 0.5 & 0.5 & 1 & 0.1 & 0.9 & 1 \\
\hline
\end{tabular}

shown in table 6. Variables are used for obtaining the overall efficiency score of DMUs. To compute the overall efficiency scores $e_{\text {ove }}$ of the $D M U \mathrm{~s}$, weighted average of the $k$ subunit scores that were obtained in stage 2 is needed, using $H_{k j}$ as defined in relation (3). Overall efficiency scores are listed in table 7. For example, for DMU 60 we have

$$
\begin{aligned}
H_{1,60}= & \delta_{1,1} x_{1,60}+\delta_{2,1} x_{2,60}+\delta_{3,1} x_{3,60}+\delta_{4,1} x_{4,60} \\
= & 0.00001 \times 452+0.00001 \times 18.97+0.00756 \\
& \times 13.17+0.00001 \times 1.114 \\
= & 0.1004 . \\
H_{2,60}= & \delta_{1,2} x_{1,60}+\delta_{3,2} x_{3,60}=0.00001 \times 452+0.06827 \\
& \times 13.17=0.8997 .
\end{aligned}
$$

Further, in order to calculate the overall efficiency of DMU 60

$$
\begin{aligned}
e_{\text {ove }_{60}} & =H_{1,60} \times \text { eff subunit } 1_{60}+H_{2,60} \times \text { eff subunit } 2_{60} \\
& =0.1004 \times 0.2818+0.8997 \times 0.2405=0.2447 .
\end{aligned}
$$

\subsection{Discussion}

Computed results are used for directing resource transfers within units. The proposed approach provides information that demonstrates how much of primary materials each production line uses, how this material is consumed and how much of it is involved in the production of pollution. Therefore, the proposed method may be used by managers in utilizing efficiency scores and related resource usage to both efficiency improvement and controlling the production of pollutants and waste produced in production lines.

As discussed in previous sections, each DMU viewed as a business unit consists of several subunits. In the suggested approach for sharing the resource in subunits, a $\beta_{i k}$ split variable is defined. This variable provides an appropriate portioning of inputs $\left(\beta_{i k} x_{i j}\right)$ to their respective subunits. It is known that sharing input is not similar to this approach in conventional DEA models affected by undesirable output.

DMUs 43 and 44 are analysed for usage of inputs and their performance as regards bad outputs. There are 4 inputs, 3 good outputs and 2 bad outputs in each DMU, and each DMU has 2 subunits as shown in table 2. For DMUs 
Table 6. $H_{k j}$ values arising from Model (4); this variable is used to obtain overall efficiency.

\begin{tabular}{|c|c|c|c|c|c|c|c|c|}
\hline$D M U_{j}$ & $H_{1 j}$ & $H_{2 j}$ & $D M U_{j}$ & $H_{1 j}$ & $H_{2 j}$ & $D M U_{j}$ & $H_{1 j}$ & $H_{2 j}$ \\
\hline 1 & 0.1002 & 0.8999 & 21 & 0.8513 & 0.1488 & 41 & 0.1019 & 0.8982 \\
\hline 2 & 0.3064 & 0.6937 & 22 & 0.1002 & 0.8999 & 42 & 0.9991 & 0.001 \\
\hline 3 & 0.807 & 0.1931 & 23 & 0.9995 & 0.0006 & 43 & 0.9976 & 0.0025 \\
\hline 4 & 0.7936 & 0.2065 & 24 & 0.2354 & 0.7647 & 44 & 0.1016 & 0.8985 \\
\hline 5 & 0.9041 & 0.096 & 25 & 0.7057 & 0.2944 & 45 & 0.8847 & 0.1154 \\
\hline 6 & 0.9009 & 0.0992 & 26 & 0.7193 & 0.2808 & 46 & 0.8555 & 0.1446 \\
\hline 7 & 0.3001 & 0.7 & 27 & 0.7074 & 0.2927 & 47 & 0.7975 & 0.2026 \\
\hline 8 & 0.1209 & 0.8792 & 28 & 0.7499 & 0.2502 & 48 & 0.9766 & 0.0235 \\
\hline 9 & 0.7961 & 0.204 & 29 & 0.9993 & 0.0008 & 49 & 0.8616 & 0.1385 \\
\hline 10 & 0.9995 & 0.0006 & 30 & 0.9989 & 0.0012 & 50 & 0.8825 & 0.1176 \\
\hline 11 & 0.9718 & 0.0283 & 31 & 0.978 & 0.0221 & 51 & 0.9992 & 0.0009 \\
\hline 12 & 0.999 & 0.0011 & 32 & 0.4732 & 0.5269 & 52 & 0.8173 & 0.1828 \\
\hline 13 & 0.8479 & 0.1522 & 33 & 0.9985 & 0.0016 & 53 & 0.8208 & 0.1793 \\
\hline 14 & 0.8938 & 0.1063 & 34 & 0.9489 & 0.0512 & 54 & 0.9995 & 0.0006 \\
\hline 15 & 0.9578 & 0.0423 & 35 & 0.9374 & 0.0627 & 55 & 0.9079 & 0.0922 \\
\hline 16 & 0.9991 & 0.001 & 36 & 0.3924 & 0.6077 & 56 & 0.9987 & 0.0014 \\
\hline 17 & 0.9991 & 0.001 & 37 & 0.8191 & 0.181 & 57 & 0.7584 & 0.2417 \\
\hline 18 & 0.1004 & 0.8997 & 38 & 0.8138 & 0.1863 & 58 & 0.9987 & 0.0014 \\
\hline 19 & 0.8346 & 0.1655 & 39 & 0.3001 & 0.7 & 59 & 0.9968 & 0.0033 \\
\hline 20 & 0.9994 & 0.0007 & 40 & 0.8238 & 0.1763 & 60 & 0.1004 & 0.8997 \\
\hline
\end{tabular}

Table 7. Subunit efficiency scores arisen from Model (1) and overall efficiency scores.

\begin{tabular}{|c|c|c|c|c|c|c|c|}
\hline DMU & Subunit 1 & Subunit 2 & Overall & DMU & Subunit 1 & Subunit 2 & Overall \\
\hline 1 & 0.3114 & 0.2951 & 0.2968 & 31 & 0.9716 & 0.7854 & 0.9676 \\
\hline 2 & 0.4576 & 0.3114 & 0.3562 & 32 & 0.8401 & 0.4947 & 0.6582 \\
\hline 3 & 0.7075 & 0.6819 & 0.7026 & 33 & 1.0000 & 1.0000 & 1.0001 \\
\hline 4 & 0.8589 & 0.5512 & 0.7954 & 34 & 1.0000 & 0.3638 & 0.9675 \\
\hline 5 & 0.5305 & 0.5641 & 0.5338 & 35 & 0.7419 & 0.2933 & 0.7138 \\
\hline 6 & 1.0000 & 1.0000 & 1.0000 & 36 & 0.6537 & 0.287 & 0.4309 \\
\hline 7 & 0.8009 & 0.4362 & 0.5457 & 37 & 0.7653 & 1.0000 & 0.8079 \\
\hline 8 & 0.8436 & 0.585 & 0.6163 & 38 & 0.4282 & 0.5444 & 0.4499 \\
\hline 9 & 0.6108 & 0.8862 & 0.6670 & 39 & 1.0000 & 0.4324 & 0.6028 \\
\hline 10 & 1.0000 & 0.829 & 1.0000 & 40 & 1.0000 & 1.0000 & 1.0000 \\
\hline 11 & 0.7111 & 0.7268 & 0.7116 & 41 & 0.8843 & 0.4468 & 0.4914 \\
\hline 12 & 0.7139 & 0.1865 & 0.7134 & 42 & 1.0000 & 0.654 & 0.9998 \\
\hline 13 & 0.4071 & 0.5171 & 0.4239 & 43 & 1.0000 & 1.0000 & 1.0000 \\
\hline 14 & 0.7038 & 0.5583 & 0.6884 & 44 & 0.5075 & 0.3047 & 0.3253 \\
\hline 15 & 1.0000 & 0.6544 & 0.9855 & 45 & 0.8385 & 0.6307 & 0.8146 \\
\hline 16 & 1.0000 & 0.5945 & 0.9997 & 46 & 0.9838 & 0.6408 & 0.9343 \\
\hline 17 & 0.9945 & 0.1983 & 0.9938 & 47 & 0.5566 & 0.3262 & 0.5100 \\
\hline 18 & 0.6506 & 0.3758 & 0.4034 & 48 & 0.3672 & 0.1552 & 0.3623 \\
\hline 19 & 0.6194 & 0.8429 & 0.6565 & 49 & 0.9453 & 0.439 & 0.8753 \\
\hline 20 & 1.0000 & 0.4332 & 0.9997 & 50 & 1.0000 & 0.6834 & 0.9629 \\
\hline 21 & 0.4800 & 0.4269 & 0.4721 & 51 & 1.0000 & 0.1943 & 0.9994 \\
\hline 22 & 0.8191 & 0.6212 & 0.6411 & 52 & 0.9215 & 1.0000 & 0.9359 \\
\hline 23 & 1.0000 & 0.454 & 0.9998 & 53 & 0.4763 & 0.3951 & 0.4618 \\
\hline 24 & 0.7619 & 0.3979 & 0.4836 & 54 & 1.0000 & 0.7148 & 0.9999 \\
\hline 25 & 1.0000 & 0.9806 & 0.9944 & 55 & 0.9448 & 0.835 & 0.9348 \\
\hline 26 & 0.3739 & 0.4207 & 0.3871 & 56 & 1.0000 & 0.3254 & 0.9992 \\
\hline 27 & 1.0000 & 1.0000 & 1.0000 & 57 & 0.7018 & 0.9926 & 0.7722 \\
\hline 28 & 0.5084 & 0.3995 & 0.4812 & 58 & 1.0000 & 0.3269 & 0.9992 \\
\hline 29 & 0.9197 & 0.2595 & 0.9193 & 59 & 0.5891 & 0.409 & 0.5886 \\
\hline 30 & 1.0000 & 0.1496 & 0.9991 & 60 & 0.2818 & 0.2405 & 0.2447 \\
\hline
\end{tabular}


43 and 44, using conventional DEA Model (2), by undesirable output the efficiency scores are obtained as 1 and 1, respectively, as shown in table 4. Aggregate efficiencies for selected intervals $\left[a_{i k}, b_{i k}\right]=[0.3,0.9]$ are obtained as 0.4454 and 1 , respectively, by employing Model (4) as shown in table 5 . It should be noted that the arbitrarily chosen intervals $a_{i k}$ and $b_{i k}$ may be any positive numbers between 0 and 1 .

For DMU 44 in table 5 and in accordance with values obtained for variable $\beta_{i k}$, subunit 1 uses $30 \%$ of input 1 , $100 \%$ of input $2,10 \%$ of input 3 and $100 \%$ of input 4 ; using Model (2), efficiency score for subunit 1 is calculated to be 0.5075 as indicated in table 7. Additionally, subunit 2 uses $70 \%$ of input 1 and $90 \%$ of input 3; utilizing Model (2), efficiency score in subunit 2 is calculated to be 0.3047 . In DMU 43, subunit 1 uses $70 \%$ of input $1,100 \%$ of input 2, $10 \%$ of input 3 and $100 \%$ of input 4; employing Model (2), efficiency score in subunit 1 is calculated to be 1 . Similarly, subunit 2 uses $30 \%$ of input 1 and $90 \%$ of input 3; using Model (2), efficiency score in subunit 2 is computed to be 1 as shown in table 7. The overall efficiencies of DMUs 43 and 44 are 1 and 0.3253 , respectively, as shown in table 7 .

Two significant items are noticed:

i One of the important features of Model (4) is that each output in this model uses input according to its requirement. As an example, in DMUs 43 and 44 and before evaluation in Model (4), efficiency of both DMUs is 1 considering bad output. DMU 43 was efficient and DMU 44 was inefficient in the case of Model (4).

ii Intervals $\left[a_{i k}, b_{i k}\right]$ that were defined for $\beta_{i k}$ may help a manager impose different sharing limits on shared input resources.

\section{Conclusions}

Recent work by Imanirad et al [27] considers a DMU as a business unit consisting of several subunits. A traditional DEA model CCR was extended in their approach to permit efficiency measurement in situations where partial inputoutput interaction exists. The term "aggregate efficiency" is suggested for measuring performance of several subunits. An important issue that should be considered in evaluating the performance of a business unit is undesirable outputs.

This work extends the current approach to take into account a business unit consisting of several subunits that produce undesirable outputs as a by-product of desirable outputs. For this purpose, the weak disposable production technology proposed by Kuosmanen [5] is used for modelling undesirable outputs. This approach may be similar to network structure DEA in resource allocation, but it is clear that these approaches are not the same. The effort presented here may be further complemented in future studies. The following are suggested efforts:
Suggestion 1: As discussed in section 3, each input $x_{i}$ is separable, but there are situations where each input $x_{i}$ may be non-separable. Model (4) may be modified to address non-separable inputs.

Suggestion 2: As described in section 3, all DMUs are assumed to have the same resource-sharing profile. However, it is possible to have a different profile for each DMU.

Suggestion 3: The proposed model may be further developed to consider non-discretionary input or output into consideration.

\section{List of Symbols}

$j \in J, j=1, \ldots, n$

$m=1, \ldots, M \quad$ set of good outputs

$s=1, \ldots, S \quad$ set of bad outputs

$i=1, \ldots, I \quad$ set of inputs

$k=1, \ldots, K$

$\mathrm{DMU}_{o}$

$v_{m j}$

$w_{s j}$

$x_{i j}$

$v_{m o}$

$w_{\text {so }}$

$x_{i o}$

$\mu_{m}$

$\theta_{s}$

$\rho_{i}$

$\beta_{i k}$

$I_{k}$

$T_{i}$

number of bundles

DMU under evaluation

$m$ th good output of $j$ th DMU

$s$ th bad output of $j$ th DMU

$i$ th input of $j$ th DMU

$m$ th good output of $\mathrm{DMU}_{o}$

$s$ th bad output of $\mathrm{DMU}_{o}$

$i$ th input of $\mathrm{DMU}_{o}$

weight for the $r$ th good output

weight for the sth bad output

weight for the $i$ th input

split variable for $x_{i j}$ in $k$ th bundle

set of inputs in $k$ th bundle

set of all $k$ bundles that have $i$ as a

member

$R_{k}\left(O_{G}, O_{B}\right)$

$\varepsilon$

$k$ th bundle that has good or bad outputs non-Archimedean and very small number

$\begin{array}{ll}H_{k j} & \begin{array}{l}\text { convex combination of } k \text { bundles } \\ \text { efficiencies }\end{array} \\ e_{\text {agg }} & \text { aggregate efficiency } \\ e_{\text {ove }} & \text { overall efficiency }\end{array}$

\section{Appendix A}

\section{Creating the input, good output and bad output bundle}

Following the notations used in Imanirad et al [27], input, good output and bad output bundles may be generated for each $k=1, \ldots, K$, where $I_{k}$ and $R_{k}\left(O_{G}, O_{B}\right)$ represent a set of inputs, and good output and bad output, respectively. These bundles are generated so that $R_{k}$ is from a mutually exclusive set and for each $k,\left(I_{k}, R_{k}\left(O_{G}, O_{B}\right)\right) \quad k=1, \ldots, K$ is maximal based on the following definition. 
Definition 1 An input, good output and bad output bundle $\left(I_{k}, R_{k}\left(O_{G}, O_{B}\right)\right)$ is considered to be maximal if it possesses the following two properties:

1. every good output $v$ and every bad output $w$ in $R_{k}\left(O_{G}, O_{B}\right)$ are influenced by every input $i$ in $I_{k}$ and no other input outside of $I_{k}$ may influence any good output $v$ or any bad output $w$ in $R_{k}\left(O_{G}, O_{B}\right)$;

2. there exists no output outside of $R_{k}\left(O_{G}, O_{B}\right)$ whose input bundle is identical to that of $R_{k}\left(O_{G}, O_{B}\right)$.

\section{Algorithm 1 (creating maximal bundles)}

Step 1: Define $\mathbf{S}$ to be an empty set.

Step 2: For each good output and bad output $(m, s)$, derive $I(m, s)$, the set of all inputs $i$ that influence $(m, s)$; transfer $I(m, s)$ to $\mathbf{S}$ and set the bundle counter as $k=1$.

Step 3: For each $I(m, s)$ in $\mathbf{S}$, compare it with every other $I\left(m^{\prime}, s^{\prime}\right)$ in $\mathbf{S}$, and identify all $I\left(m^{\prime}, s^{\prime}\right)$ that have the same input elements as $\operatorname{in} I(m, s)$. If no such $\left(m^{\prime}, s^{\prime}\right)$ is identified, create bundle $\left(I_{k}, R_{k}\left(O_{G}, O_{B}\right)\right)$ using $I(m, s)$ and $(m, s)$ so that $\quad\left(I_{k}, R_{k}\left(O_{G}, O_{B}\right)\right)=(I(m, s),(m, s))$. Remove $I(m, s)$ from $\mathbf{S}$. Go to step 4. Otherwise, group good output and bad output $(m, s)$ and all identified $\left(m^{\prime}, s^{\prime}\right)$ (having the same input sets) together to derive $R_{k}\left(O_{G}, O_{B}\right)$ and create bundle $\left(I_{k}, R_{k}\left(O_{G}, O_{B}\right)\right)$ using $I(m, s)$ and $R_{k}\left(O_{G}, O_{B}\right)$ so that $\left(I_{k}, R_{k}\left(O_{G}, O_{B}\right)\right)=(I(m, s),(m, s))$. Remove $I(m, s)$ and all identified $I\left(m^{\prime}, s^{\prime}\right)$ from $\mathbf{S}$. Go to step 4 .

Step 4: If $\mathbf{S}$ is nonempty, set $k=k+1$ and go to step 3 . Otherwise, terminate having formed the set of all bundles.

\section{References}

[1] Charnes A, Cooper W W and Rhodes E 1978 Measuring the efficiency of decision making units. Eur. J. Oper. Res. 2: 429-444

[2] Wu J, An Q, Ali S and Liang L 2012 DEA based resource allocation considering environmental factors. Math. Comput. Model. 58: 1128-1137

[3] Hailu A and Veeman T S 2001 Non-parametric productivity analysis with undesirable outputs: an application to the Canadian pulp and paper industry. Am. J. Agric. Econ. 83: 605-616

[4] Chavas J P and Cox T L 1997 Production analysis: a nonparametric time series application to U.S. agriculture. Am. J. Agric. Econ. 48: 330-348

[5] Kuosmanen T 2005 Weak disposability in non-parametric production analysis with undesirable outputs. Am. J. Agric. Econ. 87: 1077-1082

[6] Zhou Y P, Poh K L and Ang B W 2008 Measuring environmental performance under different environmental DEA technologies. Energy Econ. 30: 1-14

[7] Bain Y and Yang F 2010 Resource and environment efficiency analysis of provinces in China: a DEA approach based on Shannon's entropy. Energy Policy 38: 1909-1917
[8] Hosseinzadeh Lotfi F, Nematollahi N, Behzadi M H, Mirbolouki M and Z Moghaddasa 2012 Centralized resource allocation with stochastic data. J. Comput. Appl. Math. 236: $1783-1788$

[9] Mandal S K 2010 Do undesirable output and environmental regulation matter in energy efficiency analysis? Evidence from Indian cement industry. Energy Policy 38: 6076-6083

[10] Dakpo K H, Jeanneaux P and Latruffe L 2015 Modelling pollution-generating technologies in performance benchmarking: recent developments, limits and future prospects in the nonparametric framework. Eur. J. Oper. Res. 250: 347-359

[11] Yan H, Wei Q L and Hao G 2002 DEA models for resource reallocation and production input/output estimation. Eur. J. Oper. Res. 136: 19-31

[12] Färe R and Grosskopf S 2004 Modeling undesirable factors in efficiency evaluation: comment. Eur. J. Oper. Res. 157: 242-245

[13] Basso A and Peccati L A 2001 Optimal resource allocation with minimum activation levels and fixed costs. Eur. J. Oper. Res. 131: 536-549

[14] Hadi-Vencheh A, Foroughi A A and Soleimani-damaneh M 2008 A DEA model for resource allocation. Econ. Model. 25: 983-993

[15] Cecilio M M and Diego P 2006 On centralised resource allocation using DEA. Working Paper, Kent Business School, University of Kent, UK, pp. 146-154

[16] Gomes E G and Lins M P E 2008 Modelling undesirable outputs with zero sum gains data envelopment analysis models. J. Oper. Res. Soc. 59: 616-623

[17] Lin R C, Mustafa Y S and Pasupathy K S 2013 Multi-objective simulation optimization using data envelopment analysis and genetic algorithm: specific application to determining optimal resource levels in surgical services. Omega 41: 881-892

[18] Korhonen P and Syrjanen M 2004 Resource allocation based on efficiency analysis. Manag. Sci. 50: 1134-1144

[19] Yang T, Wang P and Li F 2018 Centralized resource allocation and target setting based on data envelopment analysis model. Math. Prob. Eng. 2018: 1-10

[20] Amirteimoori A, Toloie-Eshlaghi A and Homayoonfar M 2014 Efficiency measurement in two-stage network structures considering undesirable outputs. Int. J. Ind. Math. 6: 65-71

[21] Ming-Miin Y 2004 Measuring physical efficiency of domestic airports in Taiwan with undesirable outputs and environmental factors. J. Air Transp. Manag. 10: 295-303

[22] Fukuyama H and Weber W L 2010 A slacks-based inefficiency measure for a two-stage system with bad outputs. Omega 38: 398-409

[23] Wu J, Zhu Q, Ji X, Chu J and Liang L 2016 Two-stage network processes with shared resources and resources recovered from undesirable outputs. Eur. J. Oper. Res. 251: 182-197

[24] Mogale D G, Kumar S K and Tiwari K M 2016 Two-stage Indian food grain supply chain network transportation-allocation model. In: Proceedings of the Manufacturing IFAC Conference, pp. 1767-1772

[25] Mogale D G, Kumar S K and Tiwari M K 2018 An MINLP model to support the movement and storage decisions of the Indian food grain supply chain. Control Eng. Pract. 70: 98-113 
[26] Mogale D G, Dolgui A, Kandhway R, Kumar S K and Tiwari M K 2017 A multi-period inventory transportation model for tactical planning of food grain supply chain. Comput. Ind. Eng. 110: 379-394

[27] Imanirad R, Cook W D and Zhu J 2013 Partial input to output impacts in DEA: production considerations and resource sharing among business subunits. Nav. Res. Logist. 60: 190-207

[28] Yang H and Pollitt M 2010 The necessity of distinguishing weak and strong disposability among undesirable outputs in
DEA: environmental performance of Chinese coal-fired power plants. Energy Policy 38: 4440-4444

[29] Kuosmanen T and Podinovski V 2009 Weak disposability in nonparametric production analysis: reply to Färe and Grosskopf. Am. J. Agric. Econ. 91: 539-545

[30] Kuosmanen T and Kazemi Matin R 2011 Duality of weakly disposable technology. Omega 39: 504-512

[31] Charnes A and Cooper W W 1962 Programming with linear fractional functional. Nav. Res. Logist. 9: 181-185 